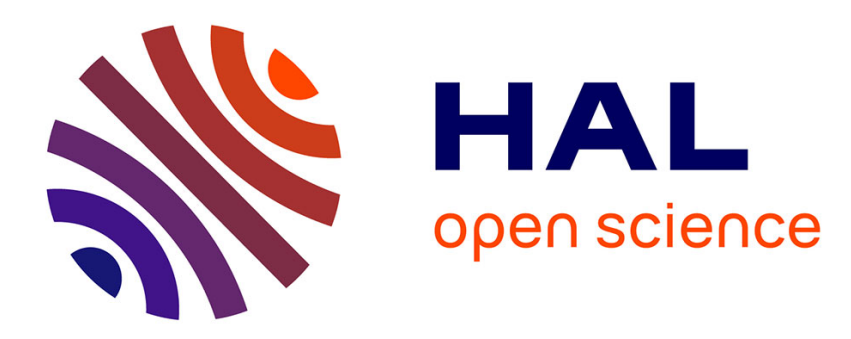

\title{
Induced Decompositions of Highly Dense Graphs
}

Nathann Cohen, Zsolt Tuza

\section{To cite this version:}

Nathann Cohen, Zsolt Tuza. Induced Decompositions of Highly Dense Graphs. Journal of Graph

Theory, 2015, 78 (2), pp.97-107. 10.1002/jgt.21792 . hal-01076882

\section{HAL Id: hal-01076882 \\ https://hal.science/hal-01076882}

Submitted on 23 Oct 2014

HAL is a multi-disciplinary open access archive for the deposit and dissemination of scientific research documents, whether they are published or not. The documents may come from teaching and research institutions in France or abroad, or from public or private research centers.
L'archive ouverte pluridisciplinaire HAL, est destinée au dépôt et à la diffusion de documents scientifiques de niveau recherche, publiés ou non, émanant des établissements d'enseignement et de recherche français ou étrangers, des laboratoires publics ou privés. 


\title{
Induced decompositions of highly dense graphs
}

\author{
N. Cohen ${ }^{*}$ Zs. Tuza ${ }^{\dagger}$
}

Latest update on 2013-12-3

\begin{abstract}
Given two graphs $F$ and $G$, an induced $F$-decomposition of $G$ is a partition of $E(G)$ into induced subgraphs isomorphic to $F$. Bondy and Szwarcfiter [J. Graph Theory, DOI: 10.1002/jgt.21654] defined the value ex* $(n, F)$ as the maximum number of edges in a graph of order $n$ admitting an induced $F$-decomposition and determined the value of $\operatorname{ex}^{*}(n, F)$ for some graphs (and families of graphs). In this paper we prove that $\operatorname{ex}^{*}(n, F)=\left(\begin{array}{l}n \\ 2\end{array}\right)-o\left(n^{2}\right)$ is valid for all graphs $F$. We also present tighter asymptotic bounds for some of the small graphs for which the exact value of ex* remains unknown. The proofs are based on the heavy use of various classes of Kneser graphs and hypergraphs.
\end{abstract}

\section{Introduction}

Given two graphs $F$ and $G$, an $F$-decomposition of $G$ is a partition of the edges of $G$ into subgraphs isomorphic to $F$. It is called an induced decomposition if the subgraphs are induced copies of $F$. Starting from Section 2 we shall only deal with the induced notion of these terms, and occasionally omit the word 'induced' hoping to not cause confusion.

*Laboratoire de Recherche en Informatique - Université Paris-Sud 11 - Rue Noetzlin, 91190 Gif-sur-Yvette, France.

${ }^{\dagger}$ Department of Computer Science and Systems Technology, University of Pannonia, Veszprém, Hungary; and Alfréd Rényi Institute of Mathematics, Hungarian Academy of Sciences, Budapest, Hungary. 
In [2], Bondy and Szwarcfiter defined the function $\operatorname{ex}^{*}(n, F)$ as the maximum number of edges in a graph on $n$ vertices that admits an induced $F$-decomposition. They determined the exact value of $\operatorname{ex}^{*}(n, F)$ (with arithmetic restrictions on $n$ in some cases) when $F$ is one of the following graphs: $C_{4}$ (the cycle of length four), $K_{1}+K_{2}$ (the graph with three vertices and one edge), $2 K_{1}+K_{2}$ (the graph with four vertices and one edge), $K_{1}+K_{1,2}$ (the graph with four vertices and two intersecting edges), $K_{1, k}$ (a star), or a complete $r$-partite graph with the same number $k$ of vertices in each part. They also proved that $\operatorname{ex}^{*}\left(n, K_{4}^{-}\right) \leq\left(\begin{array}{l}n \\ 2\end{array}\right)-\frac{n}{5}$ and $\operatorname{ex}^{*}\left(5 n, K_{4}^{-}\right) \geq\left(\begin{array}{c}5 n \\ 2\end{array}\right)-10 n$, where $K_{4}^{-}=K_{4}-e$ is the graph with four vertices and five edges.

Besides those graphs, the value of $\operatorname{ex}^{*}(n, F)$ is known for some values of $n$ when $F$ is a complete graph. Indeed, as any complete subgraph is necessarily induced, the equality $\operatorname{ex}^{*}\left(n, K_{k}\right)=\left(\begin{array}{l}n \\ 2\end{array}\right)$ is equivalent to the existence of an $(n, k, 1)$ BIBD (see [5] for such values when $k \leq 9$ ). In particular, for $k=3$, the existence of Steiner Triple Systems gives $\operatorname{ex}^{*}\left(n, K_{3}\right)=\left(\begin{array}{l}n \\ 2\end{array}\right)$ when $n \equiv 1,3(\bmod 6)$. More generally, a theorem of Wilson [18] implies that $\operatorname{ex}^{*}\left(n, K_{k}\right)=\left(\begin{array}{l}n \\ 2\end{array}\right)-O(n)$.

Theorem 1 (Wilson [18]). For any integer $k$ and sufficiently large integer $n \geq n_{k}$, the complete graph $K_{n}$ can be decomposed into edge-disjoint copies of $K_{k}$ if and only if $\left(\begin{array}{l}k \\ 2\end{array}\right)$ divides $\left(\begin{array}{l}n \\ 2\end{array}\right)$ and $k-1$ divides $n-1$.

For intermediate values of $n$, which violate the divisibility conditions, Chee et al. [4] recently proved that the number of $K_{k}$ subgraphs in a largest edge-disjoint packing is at most an additive constant $c=c(k)$ away from $\left\lfloor\frac{n}{k}\left\lfloor\frac{n-1}{k-1}\right\rfloor\right\rfloor$.

Another strong result of Wilson [17] deals with the $F$-decomposition of large complete graphs; it is important to emphasize, however, that these decompositions are not induced decompositions.

Theorem 2 (Wilson [17]). For any graph $F$ and sufficiently large integer $n$, the complete graph $K_{n}$ can be decomposed into edge-disjoint copies of $F$ if and only if $|E(F)|$ divides $\left(\begin{array}{c}n \\ 2\end{array}\right)$ and $n-1$ is divisible by the greatest common divisor of the degree sequence of $F$.

In sharp contrast to the classical extremal theory of Turán-type problems, we prove that the asymptotic equality $\operatorname{ex}^{*}(n, F)=\left(\begin{array}{l}n \\ 2\end{array}\right)-o\left(n^{2}\right)$ holds for all graphs $F$ as $n \rightarrow \infty$ (Theorem 5). Moreover, for some small graphs $F$ mentioned in [2] for which the exact value of $\operatorname{ex}^{*}(n, F)$ is not settled so far, we provide explicit constructions of dense graphs admitting induced $F$-decompositions. Those $F$ include $2 K_{2}$ (the perfect matching on 4 vertices - Theorem 3), $K_{1,3}^{+}$(the 'paw', 
i.e. a claw graph augmented with an edge), $P_{3}$ (the path of length two) and $C_{6}$ (the cycle of length six), the latter three given in Corollary 1.

The small graphs are treated in Section 2, while the general Theorem 5 is proved in Section 3.

It is important to note that several decades ago Frankl and Füredi [8] studied a very general problem on hypergraph packing, which is strongly related to the one investigated here. Given a family $\mathcal{H}$ of $t$-subsets of a $k$-set, they call a family $\mathcal{F}$ of $k$-subsets of a $v$-set a $(v, k, \mathcal{H})$-packing if for each $F \in \mathcal{F}$ there is a copy $\mathcal{H}_{F}$ of $\mathcal{H}$ such that the $t$-element sets of $F$ corresponding to $\mathcal{H}_{\mathcal{F}}$ are covered only by $F$. The main result of [8] — proved by probabilistic methods — states that, for every fixed $k$ and $\mathcal{H}$, the largest size of a $(v, k, \mathcal{H})$-packing is asymptotically $\left(\begin{array}{l}v \\ t\end{array}\right) /|\mathcal{H}|$ as $v \rightarrow \infty$.

\section{Induced decompositions into some small graphs}

In this section we give constructions of dense graphs decomposable into induced copies of the graphs $2 K_{2}, K_{1,3}^{+}, P_{3}$, and $C_{6}$.

Our constructions begin with the Kneser graphs, which are good candidates for $2 K_{2}$-decompositions. This approach is strong enough to verify the lower bound $\left(\begin{array}{l}n \\ 2\end{array}\right)-o\left(n^{2}\right)$ on $\operatorname{ex}^{*}\left(n, 2 K_{2}\right)$, nevertheless the bound obtained will be improvable by a refinement of the method. We use the standard notation $[p]:=\{1,2, \ldots, p\}$.

Definition. The Kneser graph $K G_{k}^{p}$ has the vertex set

$$
V\left(K G_{k}^{p}\right)=\left(\begin{array}{c}
{[p]} \\
k
\end{array}\right)=\{S: S \subseteq[p] \text { such that }|S|=k\}
$$

where two vertices $S, S^{\prime}$ are adjacent if and only if $S \cap S^{\prime}=\emptyset$.

Theorem 3. We have $\operatorname{ex}^{*}\left(n, 2 K_{2}\right)=\left(\begin{array}{l}n \\ 2\end{array}\right)-O\left(n^{\frac{5}{3}}\right)$.

Proof. $K G_{3}^{6}$ is a 1-regular graph with 10 edges, and therefore admits an induced $2 K_{2}$-decomposition. More generally $K G_{3}^{p}$ is a $\left(\begin{array}{c}p-3 \\ 3\end{array}\right)$-regular graph with $\frac{1}{2}\left(\begin{array}{l}p \\ 6\end{array}\right)\left(\begin{array}{l}6 \\ 3\end{array}\right)=$ $10\left(\begin{array}{l}p \\ 6\end{array}\right)$ edges - an even number - and we are going to show that $K G_{3}^{6}$ decomposes $K G_{3}^{p}$ for any $p>6$, which will yield the result.

For any 6-subset $S \subseteq[p]$, the graph $K G_{3}^{p}[S]$ induced by the vertices of $K G_{3}^{p}$ whose representative set belongs to $S$ is isomorphic to $K G_{3}^{6}$. Besides, an edge between two vertices $u=\left\{u_{1}, u_{2}, u_{3}\right\}$ and $v=\left\{v_{1}, v_{2}, v_{3}\right\}$ belongs to $K G_{3}^{p}[S]$ if and only if $S=\left\{u_{1}, u_{2}, u_{3}, v_{1}, v_{2}, v_{3}\right\}$. Hence, the $\left(\begin{array}{l}p \\ 6\end{array}\right)$ induced copies of $K G_{3}^{6}$ are 
edge-disjoint, and as $K G_{3}^{6}$ decomposes $K G_{3}^{p}$ we obtain by transitivity that $2 K_{2}$ decomposes $K G_{3}^{p}$.

When $\left(\begin{array}{l}p \\ 3\end{array}\right) \leq n<\left(\begin{array}{c}p+1 \\ 3\end{array}\right)$, we lower-bound $\operatorname{ex}^{*}\left(n, 2 K_{2}\right)$ with $\operatorname{ex}^{*}\left(\left(\begin{array}{l}p \\ 3\end{array}\right), 2 K_{2}\right)$, which leads to the result.

Decomposition results for other small graphs like $P_{3}$ or $K_{1,3}^{+}$seem to be not obtainable using this family of graphs. While there may exist an instance of $K G_{3}^{p}$ admitting a decomposition into $P_{3}$, what one would need is a way to ensure that all of them - or most of them - do so when $p$ grows large; but the technique above does not work, as the copies of $K G_{3}^{p}[S]$ would not be disjoint anymore.

We now give an alternative construction achieving precisely that, e.g. when $F$ is one of $P_{3}, C_{6}$, or $K_{1,3}^{+}$.

Definition. The Bipartite Kneser Graph $B K^{p_{1}, p_{2}}$ is defined over the set of $p_{1} p_{2}$ vertices $^{1}$

$$
V\left(B K^{p_{1}, p_{2}}\right)=\left\{v: v=\left\{v_{1}, v_{2}\right\} \text { with } v_{1} \in\left[p_{1}\right]_{1} \text { and } v_{2} \in\left[p_{2}\right]_{2}\right\}
$$

where $\left[p_{1}\right]_{1}$ and $\left[p_{2}\right]_{2}$ are disjoint copies of $\left[p_{1}\right]$ and $\left[p_{2}\right]$, and two vertices $v, v^{\prime}$ are adjacent if and only if $v \cap v^{\prime}=\emptyset$.

Remark. The graph $B K^{p_{1}, p_{2}}$ is isomorphic to $\overline{L\left(K_{p_{1}, p_{2}}\right)}$, the complement of the line graph of a complete bipartite graph on $p_{1}+p_{2}$ vertices.

We prove in Theorem 4 that a Bipartite Kneser Graph decomposes arbitrarily larger ones. This will be sufficient to obtain that $\operatorname{ex}^{*}(n, F)=\left(\begin{array}{l}n \\ 2\end{array}\right)-O\left(n^{\frac{3}{2}}\right)$ holds for $F=2 K_{2}, P_{3}, C_{6}, K_{1,3}^{+}$, as these graphs decompose small Bipartite Kneser Graphs. Indeed, $2 K_{2} \cong B K^{2,2}, P_{3}$ decomposes $C_{6}$ and $C_{6} \cong B K^{3,2}$, and $K_{1,3}^{+}$ decomposes $B K^{4,3}$ as will be shown in Lemma 1 below.

Theorem 4. The graph $B K^{p_{1}, p_{2}}$ admits an induced $B K^{a_{1}, a_{2}}$-decomposition whenever $K_{p_{1}}$ and $K_{p_{2}}$ respectively admit a $K_{a_{1}}$-decomposition and a $K_{a_{2}}$-decomposition.

Proof. Let $S_{1}$ and $S_{2}$ be respectively a subset of $\left[p_{1}\right]_{1}$ or cardinality $a_{1}$ and a subset of $\left[p_{2}\right]_{2}$ of cardinality $a_{2}$, and let $B K^{p_{1}, p_{2}}\left[S_{1}, S_{2}\right]$ denote the subgraph induced in $B K^{p_{1}, p_{2}}$ by the vertices $\left\{v=\left\{v_{1}, v_{2}\right\} \in B K^{p_{1}, p_{2}}: v_{1} \in S_{1}, v_{2} \in S_{2}\right\}$. For any choice of sets $S_{1}$ and $S_{2}, B K^{p_{1}, p_{2}}\left[S_{1}, S_{2}\right]$ is isomorphic to $B K^{a_{1}, a_{2}}$.

\footnotetext{
${ }^{1}$ Notation is meant to ensure that we can speak about $\left\{v_{1}, v_{2}\right\}$ as a set rather than an ordered pair or a vector of two components in $\left[p_{1}\right] \times\left[p_{2}\right]$.
} 
By assumption, there exists for $i=1,2$ a collection $\mathcal{C}_{i}$ of sets of size $a_{i}$ such that any two elements $v_{i}, v_{i}^{\prime}$ of $\left[p_{i}\right]_{i}$ appear together in exactly one element of $C_{i}$. We can produce an induced $B K^{a_{1}, a_{2}}$-decomposition of $B K^{p_{1}, p_{2}}$ by considering the collection $\left\{B K^{p_{1}, p_{2}}\left[S_{1}, S_{2}\right]: S_{1} \in \mathcal{C}_{1}, S_{2} \in \mathcal{C}_{2}\right\}$. Indeed, for each edge between $v=\left\{v_{1}, v_{2}\right\}$ and $v^{\prime}=\left\{v_{1}^{\prime}, v_{2}^{\prime}\right\}$ there exists exactly one set $S_{1} \in \mathcal{C}_{1}$ containing both $v_{1}$ and $v_{1}^{\prime}$, and one $S_{2} \in \mathcal{C}_{2}$ containing both $v_{2}$ and $v_{2}^{\prime}$. Therefore, the collection is the desired induced decomposition.

In particular, $2 K_{2} \cong B K^{2,2}$ decomposes $B K^{p_{1}, p_{2}}$ for any choice of $p_{1}, p_{2} \geq 2$.

Corollary 1. For every fixed $p_{1}$ and $p_{2}$ we have $\operatorname{ex}^{*}\left(n, B K^{p_{1}, p_{2}}\right)=\left(\begin{array}{c}n \\ 2\end{array}\right)-O\left(n^{3 / 2}\right)$. Moreover, by transitivity, $\operatorname{ex}^{*}(n, F)=\left(\begin{array}{c}n \\ 2\end{array}\right)-O\left(n^{3 / 2}\right)$ for $F=2 K_{2}, P_{3}, C_{6}, K_{1,3}^{+}$.

Proof. By Theorem 1, for $i=1,2$, there exists constants $c_{i}$ such that $K_{c_{i} k}$ admits a $K_{a_{i}}$-decomposition for any integer $k$. Hence, $B K^{c_{1} k, c_{2} k}$ admits an induced $B K^{a_{1}, a_{2}}$-decomposition for any integer $k$. As $k$ gets large, the number of nonedges in $B K^{c_{1} k, c_{2} k}$ grows with $n^{3 / 2}$ (where $n=c_{1} c_{2} k^{2}$ ), while the gap between $\left(c_{1} k, c_{2} k\right)$ and $\left(c_{1}(k+1), c_{2}(k+1)\right)$ adds an error term of $O(n)$ for intermediate values of $n$.

Besides, we quote from Corollary 2 of [2] that $\operatorname{ex}^{*}\left(n, 2 K_{2}\right) \leq\left(\begin{array}{l}n \\ 2\end{array}\right)-\Theta\left(n^{3 / 2}\right)$. Since $C_{6}$ decomposes into induced copies of $2 K_{2}$, this upper bound implies $\operatorname{ex}^{*}\left(n, C_{6}\right) \leq\left(\begin{array}{l}n \\ 2\end{array}\right)-\Theta\left(n^{3 / 2}\right)$, too. Hence, combining these inequalities with our Corollary 1 we obtain:

Corollary 2. We have $\operatorname{ex}^{*}\left(n, 2 K_{2}\right)=\left(\begin{array}{l}n \\ 2\end{array}\right)-\Theta\left(n^{3 / 2}\right)$ and $\operatorname{ex}^{*}\left(n, C_{6}\right)=\left(\begin{array}{l}n \\ 2\end{array}\right)-$ $\Theta\left(n^{3 / 2}\right)$.

At the end of this section we prove a lemma that completes the proof of Corollary 1 for $K_{1,3}^{+}$.

Lemma 1. The graph $B K^{4,3}$ admits an induced $K_{1,3}^{+}$-decomposition.

Proof. The following decomposition was obtained with the software Sage [16], asked to compute a maximum independent set in the graph of all induced $K_{1,3}^{+}$ subgraphs of $B K^{4,3}$, two of them being adjacent when they share an edge. It produced a list of 9 edge-disjoint graphs with 4 edges, each of them being isomorphic to $K_{1,3}^{+}$, which partitions the 36 edges of $B K^{4,3}$. 

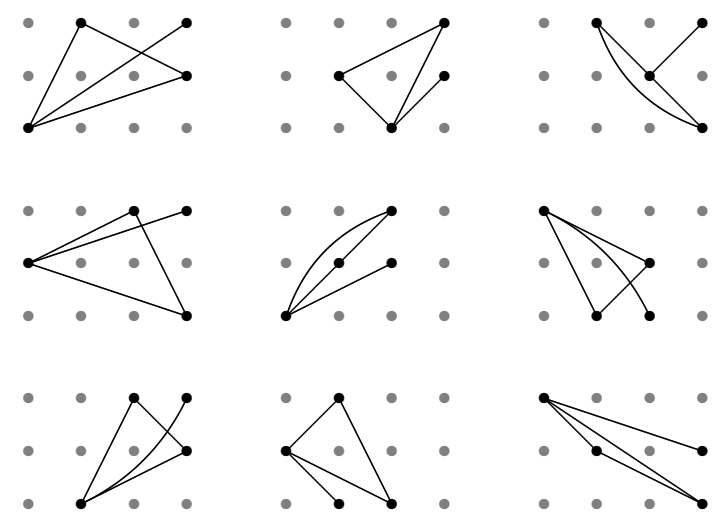

Alternatively, if we label the columns with $\{a, b, c, d\}$ and the rows with $\{1,2,3\}$ ( $1 a$ being the bottom-left corner and $3 d$ the top-right one), the decomposition is given by the graphs induced by the following sets of vertices:

$$
\begin{array}{lll}
\{1 a, 2 d, 3 b, 3 d\} & \{1 c, 2 b, 3 d, 2 d\} & \{2 c, 1 d, 3 b, 3 d\} \\
\{2 a, 1 d, 3 c, 3 d\} & \{1 a, 2 b, 3 c, 2 c\} & \{3 a, 1 b, 2 c, 1 c\} \\
\{1 b, 2 d, 3 c, 3 d\} & \{2 a, 1 c, 3 b, 1 b\} & \{3 a, 1 d, 2 b, 2 d\}
\end{array}
$$

\section{Induced decomposition into general graphs}

This section is devoted to the proof of the following general result.

Theorem 5. We have $\operatorname{ex}^{*}(n, F)=\left(\begin{array}{l}n \\ 2\end{array}\right)-o\left(n^{2}\right)$ for any graph $F$.

Let us begin with a preliminary remark. Although the family of Bipartite Kneser Graphs is sufficient to obtain decomposition results for some graphs, it cannot be hoped to provide a proof for all graphs. Indeed, Bipartite Kneser Graphs are "complements of line graphs of bipartite graphs", and for this reason none of them contains e.g. the complement of the claw graph (i.e. the graph $K_{1}+K_{3}$, cf. Fig. 1) as an induced subgraph. The second drawback comes from the method itself: in order to deduce anything on the value of the ex* function, we need to prove that a graph decomposes a Bipartite Kneser Graph, an operation which we have no specific tool for. We now enlarge the graph class once more, and prove that any graph is an induced subgraph of some graph in this family (Lemma 2). 
Definition. The Multipartite Kneser Graph $M K^{p_{1}, \ldots, p_{k}}$ is defined over the set of $\prod_{i=1}^{k} p_{i}$ vertices $^{2}$

$$
V\left(M K^{p_{1}, \ldots, p_{k}}\right)=\left[p_{1}\right]_{1} \times \cdots \times\left[p_{k}\right]_{k}=\left\{v=\left\{v_{1}, \ldots, v_{k}\right\}: v_{i} \in\left[p_{i}\right]_{i}\right\} .
$$

Two vertices of $M K^{p_{1}, \ldots, p_{k}}$ are adjacent if and only if their corresponding sets of size $k$ are disjoint. We denote by $M K^{p \times k}$ the graph $M K^{p, \ldots, p}$ on $p^{k}$ vertices (see Fig. 1).

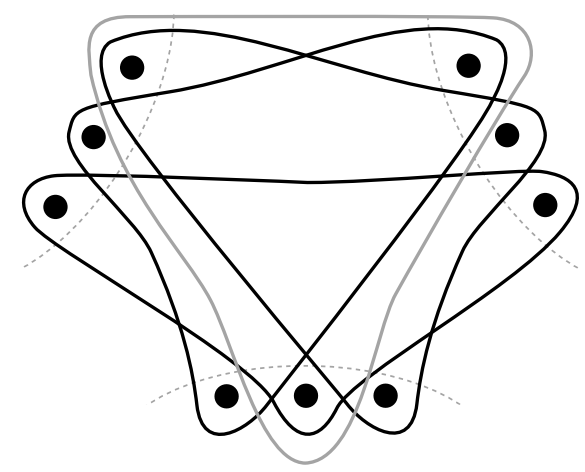

Figure 1: A realization of $K_{1}+K_{3}$ as an induced subgraph of $M K^{3 \times 3}$

Remark. As a generalization of the bipartite case, the graph $M K^{p_{1}, \ldots, p_{k}}$ is iso-

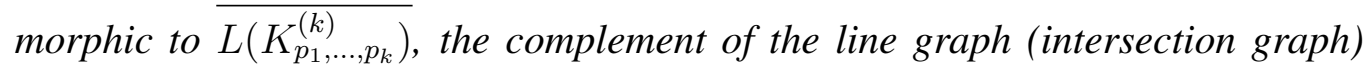
of a complete $k$-partite hypergraph whose $i^{\text {th }}$ vertex class has $p_{i}$ vertices for $i=1,2, \ldots, k$.

Lemma 2. Any graph $F$ is an induced subgraph of all $M K^{p \times k}$ for sufficiently large $p$ and $k$.

Proof. We prove that it is already sufficient to chose $p=|V(F)|$ and $k=|E(\bar{F})|$. This implies that also any larger values of $p$ and $k$ satisfy the requirements. Let us

\footnotetext{
${ }^{2}$ Notation here is analogous to that for bipartite Kneser graphs. The sets $\left[p_{1}\right]_{1}, \ldots,\left[p_{k}\right]_{k}$ are mutually disjoint copies of $\left[p_{1}\right], \ldots,\left[p_{k}\right]$, respectively. This allows us to view $v=\left\{v_{1}, \ldots, v_{k}\right\}$ as a $k$-element set rather than a $k$-tuple or a vector of length $k$. For instance, $v \cap v^{\prime}$ then then simply means intersection and not the more complicated notion of the set of coordinates in which two $k$-tuples agree.
} 
note that usually much smaller values work, too. ${ }^{3}$

Assuming that $V(F)=\{1, \ldots, p\}$ and $E(\bar{F})=\left\{e_{1}, \ldots, e_{k}\right\}$ (the set of nonedges of $F$ ), we consider the set $\left\{v^{1}, \ldots, v^{p}\right\}$ of $|V(F)|$ vertices of $M K^{p \times k}$, where

- If $i$ is an endpoint of $e_{j}=i i^{\prime}$, we set the $j$ th component $v_{j}^{i}$ of vertex $v^{i}$ to $\min \left(i, i^{\prime}\right)$ implying that $v^{i}$ and $v^{i^{\prime}}$ are not disjoint sets.

- If $i$ is not an endpoint of $e_{j}$, the $j$ th component $v_{j}^{i}$ of vertex $v^{i}$ is equal to $i$.

In such a family, two vertices $v^{i}$ and $v^{i^{\prime}}$ correspond to disjoint sets if and only if they are adjacent in $F$, ensuring that this collection of vertices induces a copy of $F$ in $M K^{p \times k}$.

Considering the symmetry properties of the $M K$ graphs, one can already expect to find many disjoints instances of $F$ inside of large instances. It is quite unlikely, though, that for a fixed $F$ all sufficiently large graphs $M K^{p \times k}$ would admit an induced $F$-decomposition, if only because of arithmetical constraints. As it is also increasingly difficult to produce $F$-decompositions of $M K$ graphs when $F$ grows large, we change our proof's methodology.

Indeed, if trying to decompose $M K^{p \times k}$ into induced copies of a graph $F$ may be too ambitious, it is actually sufficient to be able to decompose a dense subgraph of $M K^{p \times k}$ into copies of $F$ to obtain the desired result. Hence, we could be satisfied with finding many edge-disjoint induced copies of $F$ in $M K^{p \times k}$ covering most of the edges. The union of these copies is a perfectly valid example of a dense graph admitting an induced $F$-decomposition.

In order to obtain this decomposition, we will use a powerful theorem from Pippenger and Spencer [13]. Their result being much more general, its content below is specialized to suit our problem, and is similar to the version appearing in [10] (see also [9, 1]). As a matter of fact, the version in [9] is already strong enough for our purpose. For a hypergraph $\mathcal{H}$ we introduce the notation $\Delta_{2}(\mathcal{H}):=$ $\max _{x, y \in V(\mathcal{H}), x \neq y}|\{e \in E(\mathcal{H}): x, y \in e\}|$, and call it the maximum co-degree of $\mathcal{H}$. Also, if $\mathcal{H}$ is regular, we denote by $\operatorname{deg}(\mathcal{H})$ the degree of regularity.

Theorem 6 (Pippenger, Spencer [13]). Let $\mathfrak{H}$ be an infinite family of r-uniform regular hypergraphs. If

$$
\Delta_{2}(\mathcal{H}) \ll \operatorname{deg}(\mathcal{H})
$$

\footnotetext{
${ }^{3}$ It is for instance possible to chose $k=\Delta(\bar{F})+1$ and use Vizing's theorem, or to optimize on both of $p$ and $k$, or consider coverings of the edge set of $\bar{F}$ with subgraphs which are vertex-disjoint unions of complete graphs. Finding the smallest integers is probably an interesting combinatorial problem of its own.
} 
as $|V(\mathcal{H})|$ gets large (for any increasing sequence of hypergraphs $\mathcal{H} \in \mathfrak{H})$, then there exists in $\mathcal{H}$ a set of mutually disjoint hyperedges missing at most $o(|V(\mathcal{H})|)$ vertices.

This theorem can let us find the desired set of edge-disjoint induced copies of $F$ in a large $M K^{p \times k}$ if we can achieve a rephrasing of our problem in terms of a maximum hypergraph matching (a matching in an hypergraph is a set of mutually vertex-disjoint edges). What we need now is to define a hypergraph whose edges are all the induced occurrences of $F$ in $M K^{p \times k}$.

Definition. Let $\mathcal{H}^{p \times k}$ be the hypergraph whose vertex set is the set $E\left(M K^{p \times k}\right)$ of all edges of $M K^{p \times k}$, and whose hyperedges are the edge sets of all the induced subgraphs of $M K^{p \times k}$ isomorphic to $F$.

We deduce from the edge-transitivity of $M K^{p \times k}$ that the the hypergraph $\mathcal{H}^{p \times k}$ is vertex-transitive, hence regular. It is also $|E(F)|$-uniform. Besides, a matching of $\mathcal{H}^{p \times k}$ covering $(1-o(1))\left|V\left(M K^{p \times k}\right)\right|$ vertices corresponds to a collection of induced edge-disjoint copies of $F$ in $M K^{p \times k}$ covering $(1-o(1))\left|E\left(M K^{p \times k}\right)\right|$ edges, i.e. a dense subgraph of $M K^{p \times k}$ admitting an induced $F$-decomposition. Hence, to obtain Theorem 5 we need to ensure that the conditions of Theorem 6 are satisfied.

We will prove that $\Delta_{2}\left(\mathcal{H}^{p \times k}\right) \ll \operatorname{deg}\left(\mathcal{H}^{p \times k}\right)$ holds for any fixed $k>|E(\bar{F})|$ when $p$ grows large. To do so, we show that the number of induced copies of $F$ in $M K^{p \times k}$ containing two given edges is negligible compared to the number of copies containing exactly one of the two edges in question.

For the proof, let us consider two vertices of $\mathcal{H}^{p \times k}$, i.e. two edges $v_{1} v_{1}^{\prime}, v_{2} v_{2}^{\prime}$ in $M K^{p \times k}$, corresponding to four sets of size $k$. By the definition of adjacencies in $M K^{p \times k}$ we have $v_{1} \cap v_{1}^{\prime}=\emptyset$ and $v_{2} \cap v_{2}^{\prime}=\emptyset$, but $v_{1} \cup v_{1}^{\prime}$ and $v_{2} \cup v_{2}^{\prime}$ may very well intersect.

Recall that $V\left(M K^{p_{1}, \ldots, p_{k}}\right)=\left[p_{1}\right]_{1} \times \cdots \times\left[p_{k}\right]_{k}$; we shall refer to the elements of the components $\left[p_{i}\right]_{i}(1 \leq i \leq k)$ as 'points'. In our case $p_{1}=\cdots=p_{k}=p$ holds, that means $V\left(M K^{p \times k}\right)=[p]_{1} \times \cdots \times[p]_{k}$. When $k>|E(\bar{F})|$, i.e. when the size of the sets defining the vertices $v_{1}, v_{1}^{\prime}, v_{2}, v_{2}^{\prime}$ of $M K^{p \times k}$ is large compared to the number of non-edges of $F$, some of their points are not necessary to encode the adjacencies of a copy of $F$. Let us now consider a copy $F^{*} \subseteq M K^{p \times k}$ of $F$ containing the edges $v_{1} v_{1}^{\prime}, v_{2} v_{2}^{\prime}$. We are going to distinguish a set of necessary points and modify this copy by playing with the other points.

According to the definitions, any non-edge of $F^{*}$ is represented by two intersecting sets, which correspond to two vertices of $M K^{p \times k}$. If the intersection of 
those two sets is contained in $v_{1} \cup v_{1}^{\prime} \cup v_{2} \cup v_{2}^{\prime}$, we mark one point in it. Having marked (at most) one point for every non-edge of $F^{*}$, each of our four sets contains at least $k-|E(\bar{F})|$ unmarked points.

The unmarked points of $v_{1}$ and $v_{1}^{\prime}$ are not actually relevant to the adjacency properties of these sets with the other sets of the copy of $F$. Since $v_{1} \neq v_{1}^{\prime}, v_{2}, v_{2}^{\prime}$, we can obtain a different induced copy of $F$ containing $v_{2} v_{2}^{\prime}$ but avoiding $v_{1} v_{1}^{\prime}$ by replacing in $v_{1}$ these unmarked points by any other point not contained in $F^{*}$, i.e. at least $p-|V(F)|$ alternatives for each of the $k-|E(\bar{F})|$ unmarked points.

As a conclusion, one can associate to any copy $F^{*}$ containing the edges $v_{1} v_{1}^{\prime}$ and $v_{2} v_{2}^{\prime}$ a set $f\left(F^{*}\right)$ of at least $(p-|V(F)|)^{k-|E(\bar{F})|}$ copies of $F$ using $v_{2} v_{2}^{\prime}$ but not $v_{1} v_{1}^{\prime}$ (and differing from $F^{*}$ only by the content of $v_{1}$ ). Furthermore, at most $c_{F}$ choices of $F^{*}$ can produce a given alternative copy of $F$, where $c_{F}$ is a constant depending on $F$ but not on $p$. (To reverse the operation, it is enough to identify one set of the copy and replace it with $v_{1}$ or $v_{1}^{\prime}$.)

We deduce that in $\mathcal{H}^{p \times k}$ the degree of a vertex is at least $\Theta\left(p^{k-|E(\bar{F})|}\right) \geq \Theta(p)$ times more than the co-degree of the pair $v_{1} v_{1}^{\prime}, v_{2} v_{2}^{\prime}$ (chosen arbitrarily), yielding $\Delta_{2}\left(\mathcal{H}^{p \times k}\right) \ll \operatorname{deg}\left(\mathcal{H}^{p \times k}\right)$ as $p$ grows large. Thus, Theorem 6 implies the existence of a family of edge-disjoint induced copies of $F$ in $M K^{p \times k}$ covering almost all edges of $M K^{p \times k}$ as $p$ gets large. Now the proof is completed by the observation that $M K^{p \times k}$ has $p^{k}$ vertices and is regular of degree $(p-1)^{k}=p^{k}-O\left(p^{k-1}\right)-$ the number of sets disjoint from a given set in $[p]_{1} \times \cdots \times[p]_{k}$ - therefore almost all vertex pairs are adjacent in $M K^{p \times k}$.

\section{Conclusion}

We have proved that the largest graphs of order $n$ admitting an edge decomposition into induced copies of a given non-edgeless graph $F$ have $\left(\begin{array}{l}n \\ 2\end{array}\right)-o\left(n^{2}\right)$ edges for each graph $F$ as $n$ gets large. This result solves the problem of Bondy and Szwarcfiter completely in the asymptotic sense. Viewing it from the other side, however, it just opens the field for a new track of studies:

Problem 1. Given a graph $F$, determine the exact or asymptotic value of

$$
\left(\begin{array}{l}
n \\
2
\end{array}\right)-\mathrm{ex}^{*}(n, F)
$$

as a function of $n$. 
This question also appears as Problem 2 in [2]. Some of our results also give a tight answer, apart from a multiplicative constant, to this formulation, exhibiting graphs $F$ with $\left(\begin{array}{l}n \\ 2\end{array}\right)-\operatorname{ex}^{*}(n, F)=\Theta\left(n^{3 / 2}\right)$. Some more cases are settled in the recent paper [11], solving Problem 1 of [2] as a corollary. Substantially different examples, with linear growth, are $K_{4}^{-}$, the stars, $C_{4}$ and more generally the complete equipartite graphs in [2], and further infinite families in [11] and [3]. It is worth noting that, although the order of magnitude of $\left(\begin{array}{l}n \\ 2\end{array}\right)-\operatorname{ex}^{*}(n, F)$ is determined in those cases, still there are very few graphs for which the exact value (as a function of $n$ ) is known.

Many problems of interest remain open. Some have been raised at the end of [2]; here we mention further ones.

Conjecture 1. For every $F$ there exist positive reals $c_{1}, c_{2}$ and a rational $\alpha(1<$ $\alpha \leq 2)$ such that

$$
c_{1} n^{\alpha} \leq\left(\begin{array}{l}
n \\
2
\end{array}\right)-\operatorname{ex}^{*}(n, F) \leq c_{2} n^{\alpha}
$$

holds for all $n \geq|V(F)|$.

Conjecture 2. For every $F$, with the value $\alpha$ defined in Conjecture 1, the sequence $\left(\left(\begin{array}{l}n \\ 2\end{array}\right)-\mathrm{ex}^{*}(n, F)\right) / n^{\alpha}$ has only finitely many limit points.

Note that more than one limit point can exist; for example if $F=C_{4}$ then there are two of them, one for $n$ even and one for $n$ odd.

Problem 2. What is the expected asymptotic growth $f_{t}(n)$ of $\left(\begin{array}{l}n \\ 2\end{array}\right)-\operatorname{ex}^{*}(n, F)$ as a function of $n$ if $F$ is chosen at random from the graphs on $t$ vertices while $n \gg t$ but the parameter $t$ also gets large?

An upper bound can be derived from the principles of our construction. One can observe that the value $k$ in the multipartite Kneser graph $M K^{p \times k}$ does not need to be larger than 1 plus the minimum $k^{\prime}$ such that the edge set of the complement $\bar{F}$ of $F$ can be covered with $k^{\prime}$ complete subgraphs.

In the case where $F$ is a complete graph, Problem 1 asks exactly about the smallest possible size of the leave graph of a partial Steiner system. Several related results are available for triple systems. The smallest leave of an $\operatorname{STS}(n)$ was determined by Schönheim [14] and Spencer [15]. Further relatively small graphs which are leave graphs of some $\operatorname{STS}(n)$ were described in $[6,12]$. For a wealthy collection of results on leave graphs, we refer to Section 9 of [7]. 


\section{Acknowledgments}

The authors thank Adrian Bondy for helpful discussions and Jean-Claude Bermond for his careful readings. This work has been partially supported by EA EWIN, ANRs AGAPE and GRATEL and by the ESF EUROCORES programme EuroGIGA, CRP ComPoSe, Fonds National de la Recherche Scientifique (F.R.S.FNRS). Research of the second author was supported in part by the Hungarian Scientific Research Fund, OTKA grant 81493, and by the Hungarian State and the European Union under the grant TAMOP-4.2.2.A-11/1/KONV-2012-0072.

\section{References}

[1] N. Alon and R. Yuster. On a hypergraph matching problem. Graphs Combin., 21(4):377-384, 2005.

[2] J. A. Bondy and J. L. Szwarcfiter. Induced decompositions of graphs. J. Graph Theory, 72:462-477, 2013.

[3] Cs. Bujtás and Zs. Tuza. Transversal designs and induced decompositions of graphs. Manuscript, in preparation.

[4] Y. M. Chee, C. J. Colbourn, C. H. Ling, and R. M. Wilson. Covering and packing for pairs. J. Combin. Theory Ser. A, 120:1440-1449, 2013.

[5] C. J. Colbourn and J. H. Dinitz, editors. Handbook of Combinatorial Designs. CRC Press, 2006.

[6] C. J. Colbourn and E. S. Mahmoodian. Support sizes of sixfold triples systems. Discrete Math., 115:103-132, 1993.

[7] C. J. Colbourn and A. Rosa. Triple Systems. Clarendon Press, 1999.

[8] P. Frankl and Z. Füredi. Colored packing of sets. Ann. Discrete Math., 34:165-178, 1987.

[9] P. Frankl and V. Rödl. Near perfect coverings in graphs and hypergraphs. European J. Combin., 6:317-326, 1985.

[10] David A. Grable. More-than-nearly-perfect packings and partial designs. Combinatorica, 19(2):221-239, 1999. 
[11] V. Halász and Zs. Tuza. Asymptotically optimal induced decompositions. Manuscript, 2013.

[12] S. Milici and Zs. Tuza. Coverable graphs. Studia Sci. Math. Hungar., 30:329-344, 1995.

[13] N. Pippenger and J. Spencer. Asymptotic behavior of the chromatic index for hypergraphs. J. Combin. Theory Ser. A, 51(1):24 - 42, 1989.

[14] J. Schönheim. On maximal systems of $k$-tuples. Studia Sci. Math. Hungar., 1:363-368, 1966.

[15] J. Spencer. Maximal consistent families of triples. J. Combin. Theory, 5:1-8, 1968.

[16] W. A. Stein et al. Sage Mathematics Software (Version 4.6.2). The Sage Development Team, 2010. http: / /www. sagemath.org.

[17] R. M. Wilson. Decomposition of complete graphs into subgraphs isomorphic to a given graph. Congr. Numer., XV:647-659, 1975.

[18] R. M. Wilson. An existence theory for pairwise balanced designs, III: Proof of the existence conjectures. J. Combin. Theory Ser. A, 18(1):71-79, 1975. 